\title{
Tecnología y sociedad: ¿Por qué no llega el hogar digital?
}

\section{Technology and society: What hinders the arrival of the digital home?}

\author{
G. Cano ${ }^{(*)}$, J. M. Maestre ${ }^{(*)}$
}

\section{RESUMEN}

Se analizan los motivos que lastran el despegue del hogar digital a través del estudio de la evolución de la tecnología y los cambios demográficos y de vivienda a lo largo de la última década, contrastando los pronósticos realizados en la época con la compleja realidad que finalmente aconteció. Durante este periodo, el sector inmobiliario experimentó uno de los mayores periodos de crecimiento de las últimas décadas y, sin embargo, la innovación en materia tecnológica apenas fue significativa en esta industria. Los cambios en la composición de las familias y su mayor apertura cultural sobre nuevas formas de habitar también abrieron posibilidades a un mercado domótico que no termina de insertarse en la vivienda española. Se plantean algunas claves explicativas y opciones de futuro.

Palabras clave: Domótica; hogar digital; vivienda; sociedad.

\section{ABSTRACT}

In this work we analyze the reasons that hinder the arrival of the digital home. To this end, we study the evolution of technology and demographic and housing changes over the last decade, contrasting the forecasts made at the time with the complex reality that finally happened. During this time, the real estate sector experienced one of its greatest growth periods, but, paradoxically, the technological innovation was barely significant in this industry. The changes in the composition of families together with a greater cultural openness towards new ways of inhabiting also brought new opportunities to the home automation market, which so far has not been capable to achieve a significant success. Finally, we provide some explanatory keys and future prospects.

Keywords: Domotics; digital home; housing; society.

(*) Universidad de Murcia (España).

(*) Universidad de Sevilla (España).

Persona de contacto/Corresponding author: pepemaestre@us.es (J. M. Maestre)

Cómo citar este artículo/Citation: Cano, G., Maestre, J. M. (2015). Tecnología y sociedad: ¿Por qué no llega el hogar digital? Informes de la Construcción, 67(538): eo9o, doi: http://dx.doi.org/10.3989/ic.13.154.

Licencia / License: Salvo indicación contraria, todos los contenidos de la edición electrónica de Informes de la Construcción se distribuyen bajo una licencia de uso y distribución Creative Commons Reconocimiento no Comercial 3.o. España (cc-by-nc). 


\section{INTRODUCCIÓN}

En estos últimos años, nuevos dispositivos tecnológicos han invadido nuestra vida hasta hacerse imprescindibles, pero cabe preguntarse hasta qué punto han cambiado también nuestro hogar. En una época en la que la casa del futuro puede empezar a hacerse realidad tal y como ha sido popularizada en los relatos de ciencia ficción, la difusión de este tipo de hogares tecnológicos no es tan rápida como se esperaba. Sin embargo, otros componentes fundamentales involucrados en la vivienda, la familia y la construcción, han experimentado grandes cambios. Sin duda, la relación entre tecnología y sociedad se torna cada vez más compleja, protagonizando esta última un papel destacado en la configuración, diseño y difusión de los procesos tecnológicos. En este sentido parece hoy más claro que nunca que tecnología y sociedad se co-producen continuamente (1).

En este artículo se estudian paralelamente la evolución de la tecnología y la vivienda con el objeto de analizar los motivos que lastran el despegue del hogar digital. Los cambios en la composición de las familias y su mayor apertura cultural sobre nuevas formas de habitar, abren posibilidades a un mercado domótico que no termina de insertarse en la vivienda española. Por su parte, el sector inmobiliario ha experimentado uno de los mayores periodos de crecimiento de las últimas décadas modificando el panorama económico y, sin embargo, la innovación en materia tecnológica apenas ha sido significativa en esta industria. Es evidente que la crisis económica en general, y la del sector inmobiliario en particular, ha contribuido a frenar todos los escenarios futuros que se habían dibujado con respecto a la implantación de los sistemas domóticos, pero es necesario analizar qué otros factores confluyen en esta situación y qué opciones de futuro pueden esperarse.

\section{TECNOLOGÍA Y HOGAR}

A través de estudios de mercado es posible caracterizar el sector domótico tanto a nivel de público objetivo como de oferta de productos y servicios. No obstante, la constante evolución de la tecnología y la histórica falta de un consenso acerca de los requisitos para que un hogar sea considerado como digital, obligan a tomar los diferentes estudios de mercado con cierta cautela. Por ejemplo, estudios como los realizados por Berg Insight (2) se centran únicamente en dispositivos que cuentan con una aplicación o portal web para su control, esto es, no consideran como parte del hogar digital aquellos dispositivos controlables únicamente mediante interruptores o mandos a distancia. Otras definiciones empleadas van más encaminadas hacia la prestación de servicios. Por ejemplo, el Real Decreto 346/2011, que desarrolla el reglamento regulador de las infraestructuras comunes de telecomunicaciones para el acceso a los servicios de telecomunicación en el interior de las edificaciones, define el hogar digital como «el lugar donde las necesidades de sus habitantes, en materia de seguridad y control, comunicaciones, ocio y confort, integración medioambiental y accesibilidad, son atendidas mediante la convergencia de servicios, infraestructuras y equipamientos». Como es lógico, diferentes definiciones de domótica y hogar digital conducen a diferentes datos con respecto al mercado, si bien todos ellos proporcionan una indicación con respecto al estado y evolución del hogar digital.

Según este mismo estudio de Berg Insight (2), el líder mundial en el desarrollo del hogar digital es EE.UU., donde se al- canzaron en 2012 los 2,9 millones de instalaciones, lo que se correspondería con una penetración de en torno al $3 \%$. La situación en Europa muestra un retraso de 3 años con respecto al mercado americano, con una base de 0,93 millones de hogares digitales en 2012, lo que corresponde a una penetración del o,4 \%. A nivel europeo, España es el quinto mercado por volumen de negocio relacionado con productos del hogar digital con una cuota del $5 \%$ (3). Los países a la cabeza son Alemania, Francia y Reino Unido con un $47 \%$, 10 \% y 8 \% respectivamente.

El mercado español de la domótica quedó particularmente bien retratado durante los años de la burbuja inmobiliaria. Diversas instituciones y empresas realizaron estudios de mercado que nos permiten analizar la evolución de las expectativas generadas en torno al mismo. La mayoría de ellos pronosticaban con optimismo la implantación de los sistemas de domótica en el periodo expansivo de la construcción registrado hasta 2007, y si bien puede decirse que se produjo un aumento de la proporción de las viviendas con sistemas domóticos - hasta el 8,25 \% de implantación en obra nueva en 2007 (4) -, la penetración final de la domótica no fue la esperada.

\subsection{Evolución de la tecnología}

La domótica consiste en la aplicación de técnicas de automatización al hogar. Como cualquier rama de la técnica relacionada con la informática y las comunicaciones, ha sufrido una fuerte evolución desde sus comienzos hasta la actualidad.

El primer sistema domótico que tuvo éxito desde un punto de vista comercial fue X10, que fue creado a finales de los años 70 por la empresa escocesa Pico Electronics. Pese a su antigüedad, se trata de un sistema que todavía hoy es utilizado por su bajo precio y su facilidad de instalación. Los servicios ofrecidos por X10 están relacionados con lo que tradicionalmente se ha entendido como domótica: una serie de automatismos básicos que permiten programar y telecontrolar el funcionamiento de enchufes, luces o persianas.

A medida que las telecomunicaciones y las redes de ordenadores se desarrollaban, las posibilidades ofrecidas por la domótica se multiplicaron. Durante los años 90, aparecieron sistemas como EIB (European Installation Bus) y Lonworks (5), que sistematizaban la introducción de instalaciones domóticas en grandes instalaciones como edificios.

La revolución propiciada por los avances en las tecnologías de las comunicaciones y por la generalización del uso de internet también tuvo su impacto en la domótica. Muestra de ello es la aparición del concepto de hogar digital (6), en el que los automatismos domóticos constituyen solo uno de los servicios que la tecnología brinda a sus usuarios. Como se ha comentado, bajo el paraguas del hogar digital se habla de servicios en materia de comodidad, seguridad, eficiencia energética, multimedia y comunicaciones. Estos avances se vieron reflejados en una nueva generación de sistemas domóticos en los que la domótica y las comunicaciones se entrelazaban para ofrecer nuevos servicios al usuario.

Precisamente, el punto álgido de la burbuja inmobiliaria fue uno de los momentos en los que muchos analistas apuntaban a que el despegue de este mercado era inminente. Esta sensación generalizada queda contrastada por diferentes ini- 
ciativas puestas en marcha durante la época. Por ejemplo, no es casual que el primer Máster de Domótica y Hogar Digital impartido en España por la Universidad Politécnica de Madrid tuviera su primera edición en 2004, como tampoco lo es que en ese mismo año tuviera su primera edición Domogar, una feria tecnológica dedicada en exclusiva a la promoción del hogar digital. Incluso en los años en los que el mercado empezaba a mostrar los primeros signos de flaqueza se esperaba que la domótica triunfara como un elemento de diferenciación dentro de la oferta inmobiliaria que permitiría hacer frente a la previsible contracción del sector.

El entusiasmo reinante fue un aliciente para la creación de empresas relacionadas con la domótica, aunque desde un punto de vista tecnológico no se introdujo ningún progreso verdaderamente significativo hasta la llegada del iPhone en 2007 y iPad en 2010. Estos dos dispositivos introdujeron nuevas cotas de usabilidad y hoy en día cualquier sistema domótico ofrece interfaz de telecontrol a través de móvil o tableta. Anteriormente, el control y la programación de la domótica se realizaba de muy diversas formas con resultados de usabilidad desiguales: televisor, ordenador, mandos a distancia, pantallas encastradas en la pared... No es de extrañar, por tanto, que algunos estudios de mercado, como los de García Montalvo (7), ya apuntaran la necesidad de una mayor accesibilidad de los sistemas, así como de una mayor compatibilidad entre marcas que facilitara el uso.

A pesar de que la dirección apuntada por los análisis de mercado era correcta, el perfeccionamiento de los servicios existentes y el desarrollo de nuevos terminales inteligentes no fue lo suficientemente rápido para evitar el brusco ajuste que vivió el sector de la construcción a partir de 2007, tal y como puede observarse en la Figura 1, que muestra la evolución del número de licencias para viviendas de nueva planta y el número de instalaciones domóticas en viviendas de nueva construcción. La magnitud del ajuste fue tan acusada como para que no hubiera posibilidad de que la domótica sirviera como elemento de diferenciación en un mercado inmobiliario en contracción, tal y como apuntaban algunos analistas.
Finalmente, otro aspecto clave desde el punto de vista tecnológico es que la mayoría de sistemas domóticos son de tipo cableado, es decir, exigen el tendido de un cableado específico en la vivienda. En el caso de una vivienda de nueva construcción, esto no supone un problema, pero para viviendas ya construidas se necesita realizar una obra, con la inversión y las molestias que implica. Por ello, hasta el momento la salud del sector ha mostrado una fuerte dependencia con respecto al número de viviendas de nueva construcción, por lo que no es de extrañar el rápido contagio tras el pinchazo de la burbuja inmobiliaria.

\subsection{Evolución de la vivienda}

El sector de la construcción tiene mucho que decir respecto a la implantación de la domótica en España. Como es lógico, en una etapa en la que se ha llegado a triplicar, en relación a la población, la media europea de construcción de viviendas (8), también se han disparado el número de instalaciones domóticas. Por otra parte, la especial sensibilidad a este sector, no ya del mercado domótico, sino del conjunto de la economía española, la convierte en bastante susceptible de los ciclos constructores, tal y como se ha hecho evidente en estos años de burbuja y pinchazo inmobiliario (9) (7). Esta estrecha relación entre construcción y domótica se percibe con claridad en la Figura 1, en la que se representa con línea continua la evolución temporal del número de licencias de viviendas de nueva construcción y con línea discontinua el número de viviendas construidas con algún tipo de instalación domótica (10) (5) (4).

En este sentido, el papel de la regulación es clave para que la construcción contribuya de forma definitiva a la implantación de la domótica. En materia legislativa ha habido una evolución desde las primeras normativas sobre mínimos de seguridad y de salud de instalaciones de sistemas domóticos en los edificios (Real Decreto 1627/1997 de 24 de octubre) hasta una posterior regulación de los sistemas que se iban incluyendo en materia de vivienda como reglamento electrónico (Real Decreto 842/2002, de 2 de agosto), compatibilidad

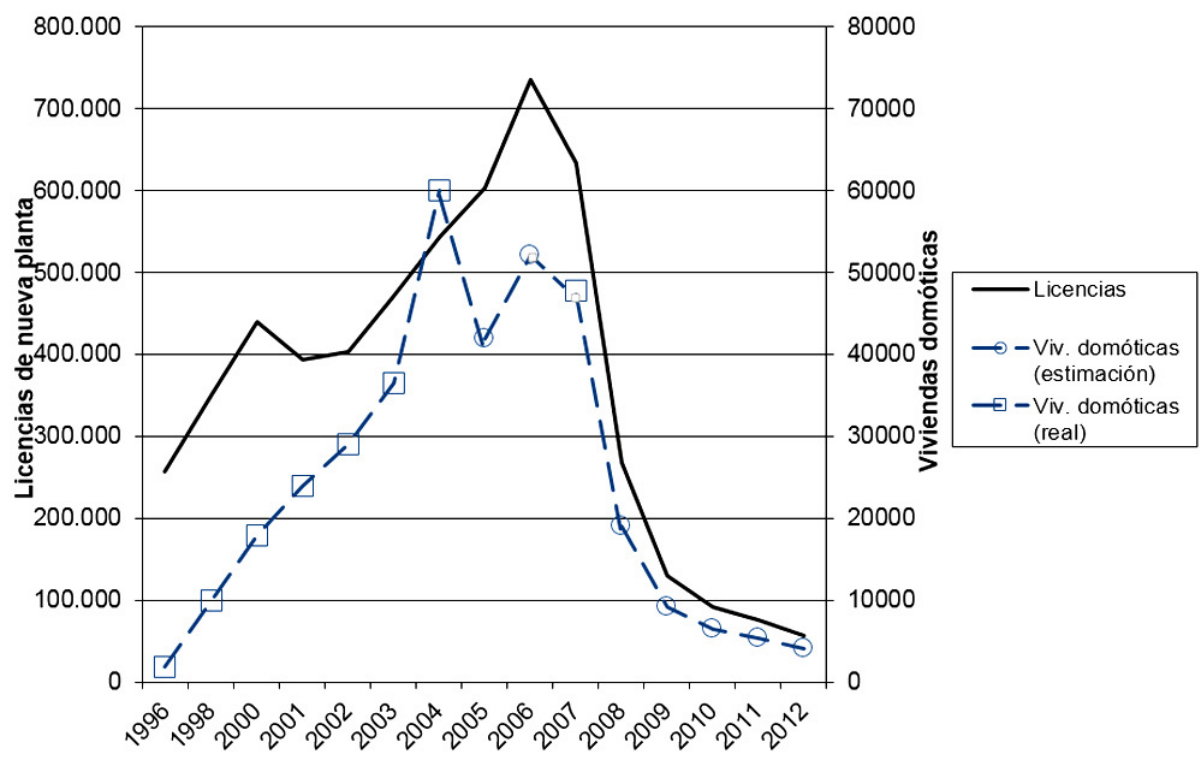

Figura 1. Licencias. $N^{0}$ de viviendas de nueva planta en edificaciones residenciales destinadas a vivienda familiar (línea continua, eje izquierdo en la figura) y número de instalaciones domóticas en viviendas de nueva construcción (línea discontinua, eje derecho en la figura). 
electromagnética de equipos radio-electrónicos y equipos terminales de telecomunicaciones (Real Decreto 1890/2000, de 20 de noviembre). La normativa más específica de recomendaciones sobre Hogar Digital queda recogida en el Anexo V del Real Decreto 346/2011.

Lamentablemente, la llegada de este último real decreto fue tardía. Sin duda, una normativa de esta naturaleza hubiera tenido un impacto muy notable una década antes, cuando la actividad del sector inmobiliario se encontraba en plena efervescencia. En ese momento, la regulación hubiera sido clave para establecer unas directrices comunes conforme a lo que un hogar digital debería ofrecer a sus ocupantes.

Por otro lado, en este periodo de gran expansión y posterior decrecimiento inmobiliario también llevó a cambios en las características de la propia vivienda. Las nuevas construcciones iniciadas han seguido pautas muy diferentes antes y después de los años de mayor apogeo inmobiliario. Las licencias según tipo de obra destinadas a vivienda familiar, nos muestran un cambio de tendencia en la construcción de viviendas unifamiliares ya que descendieron su importancia proporcional sobre el número total de viviendas frente a las viviendas no unifamiliares durante los años de mayor número de licencias expedidas, pasando, después de 2007 a protagonizar un incremento considerable de su proporción a nivel nacional, incluso por encima de los porcentajes registrados en el 2000, tal y como aparece en la Figura 2, aunque en 2012 esta tendencia parece descender.

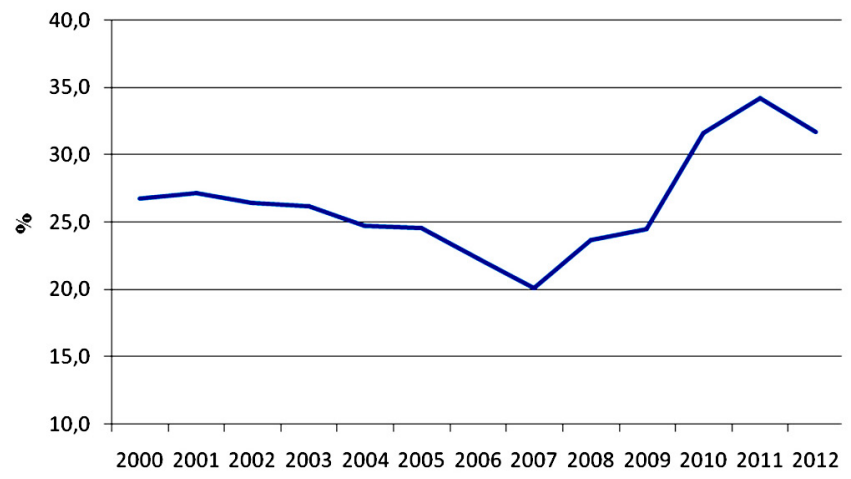

Figura 2. Porcentaje de licencias de obra expedidas de vivienda unifamiliar sobre el total de viviendas destinadas a vivienda familiar. Fuente: Elaboración propia a partir de datos del Ministerio de Fomento (Series Estadísticas).

Dentro de este incremento en la proporción de viviendas unifamiliares encontramos que no todos los tipos de viviendas aumentan por igual. Dentro de la clasificación de viviendas unifamiliares se pueden encontrar tres tipos: adosadas, pareadas o aisladas. La evolución de las mismas no ha sido homogénea, encontrando que son las viviendas aisladas las que muestran un incremento mayor en el periodo 2007-2012, y las pareadas las que menos crecen a nivel nacional. En este tipo de viviendas, la aplicación de unos sistemas de domótica de mayor nivel puede plantearse como una opción factible.

A pesar de que la mayoría de estudios de mercado no analizan a este nivel de profundidad el mercado inmobiliario, es evidente que el seguimiento de este tipo de cambios en el sector de la construcción puede ayudar a dirigir la oferta con mayor acierto.

\subsection{Evolución demográfica y de la sociedad}

Los cambios demográficos y de estructura de los hogares, aunque se han demostrado insuficientes para explicar la magnitud del fenómeno de expansión inmobiliaria en los primeros años del siglo XXI (11), conforman una parte ineludible de los factores de evolución de la vivienda. Desde el sector de la domótica estos cambios se convierten en retos y potencialidades, ya que plantean nuevas necesidades y nuevos usuarios con perfiles muy diferenciados.

Muchos de los estudios de mercado (10) (5) ya previeron escenarios demográficos como el envejecimiento de la población o el menor número de hijos por mujer, así como el aumento de los hogares unipersonales. Sin embargo, no parece que esto sirviera para motivar la adquisición de sistemas en los nuevos hogares o en los ya existentes en la proporción que se esperaba. Las nuevas necesidades derivadas de un cambio en la tipología y composición de los hogares no se tradujeron, de manera directa, en una mayor demanda de sistemas domóticos, lo que nos devuelve a la complejidad de las dinámicas de estos años y de las propias previsiones realizadas.

\section{FRENOS DEL SECTOR DOMÓTICO}

Tras el estudio de los pilares fundamentales sobre los que se apoya el mercado domótico y su pertinente análisis, estamos en condiciones de señalar algunos de los frenos del sector domótico:

1. La contracción del mercado inmobiliario fue muy abrupta. Las viviendas dejaron de venderse, ya fuera con domótica o sin ella, por lo que la demanda se desplomó (véase la Figura 1).

2. Las expectativas asociadas al valor que los usuarios darían a sus instalaciones domóticas estaban sobredimensionadas, lo cual no es de extrañar por la propia dinámica de la burbuja. En efecto, los análisis de mercado acertaron plenamente al señalar el incremento de los hogares unipersonales, pero se equivocaron al asumir que esto se traduciría en un incremento de instalaciones basándose en una supuesta facilidad de mantenimiento de un hogar domótico frente a otro que no lo es. Además, el ahorro de tiempo derivado del uso de sistemas domóticos es discutible también. Históricamente, las tareas domésticas cuyo tiempo de realización ha disminuido como consecuencia de un progreso técnico han pasado a realizarse con mayor frecuencia (12), lo que cuestiona el consiguiente ahorro de tiempo.

3. El elevado precio de muchos de los sistemas ha contribuido a que la instalación de domótica en los hogares haya quedado reservada a los tecnófilos. Asimismo, la fiabilidad de las soluciones autoinstalables ha sido baja hasta hace poco tiempo, requiriendo además considerables conocimientos por parte del usuario, lo que no ayuda a la expansión del mercado. De algún modo, la finalidad última de estos sistemas, que no es otra que satisfacer necesidades humanas (12), queda oscurecida por estos problemas.

4. En la burbuja, el precio de las viviendas, lejos de moverse en los estándares económicos clásicos, respondía a comportamientos sin base económica lógica (13). Dado que la tecnología no ha demostrado ser un factor competitivo en 
el contexto de la promoción inmobiliaria, los mayores precios no se tradujeron en una mejor dotación tecnológica.

5. El concepto de domótica y hogar digital no estuvo bien definido desde un principio. Durante algunos años de la burbuja la mera instalación de un termostato ya hacía publicitar las viviendas como domóticas (14).

6. Hasta la fecha, el mercado domótico siempre ha sido dirigido por la oferta (15), esto es, la demanda nunca ha sido lo suficientemente importante como para que los fabricantes diseñen sus dispositivos teniendo en cuenta lo que funciona bien comercialmente. La realidad es que hasta el momento la domótica no ha encontrado una aplicación que la convierta en un componente imprescindible dentro de una vivienda. Por ejemplo, la cantidad de interfaces de control existentes hasta la consolidación de los teléfonos inteligentes y las tabletas refleja con claridad esta falta de éxito dentro del mercado de masas.

7. Para que los usuarios finales perciban claramente el valor de la domótica y quieran instalarla en sus hogares, es necesario conectar con sus necesidades reales. En este sentido, los resultados de Mint-Casadomo (4) acerca de la valoración que los usuarios dan a los servicios domóticos son reveladores. En particular, la prestación más valorada es la del incremento de seguridad como consecuencia de la instalación de alarmas técnicas (por ejemplo, humo, incendio, agua, o gas). La segunda posición es para la teleasistencia, servicio fundamental en caso de que haya personas dependientes en el hogar. Cierran el podio los servicios relacionados con la telefonía, la banda ancha y la televisión. En la parte baja de la tabla aparecen los sistemas de acceso biométricos, las cámaras en el interior de la vivienda y los electrodomésticos inteligentes. Como puede verse, hay servicios tradicionalmente asociados a la domótica que no aparecen entre los mejor valorados. Por contra, uno de los más recurrentes en la mente del consumidor - el de los electrodomésticos inteligentes - se encuentra entre los peor valorados. Todo esto nos permite detectar un problema capital: existe una falta de correspondencia notable entre los servicios mejor valorados por parte de los usuarios y las expectativas del consumidor con respecto al potencial de una instalación domótica. Como es lógico, un consumidor difícilmente se interesaría por una tecnología de la que casi no espera obtener ningún servicio útil. La única forma de resolver este problema es la pedagogía. Los agentes principales del sector deben movilizarse para corregir la idea que los consumidores tienen acerca de la domótica hasta eliminar la falta de sintonía entre realidad y expectativas.

8. El estudio de Mint-Casadomo (4) indica que los sistemas domóticos más encontrados en el mercado en 2007 fueron $\mathrm{KNX} / \mathrm{EIB}(20 \%$ ) y X10 (20 \%). A partir de ahí destacan Lonworks (9 \%), EHS (9 \%), CAN (9 \%), X2D (8 \%) y Zigbee (4\%). Entre todos, no cubren el $70 \%$ de instalaciones, lo que indica la ausencia de un dominador claro en el mercado. Por tanto, se aprecia con claridad la necesidad de interoperabilidad y estandarización del sector. No hay ningún mercado bien consolidado que permita la coexistencia de tantos sistemas diferentes incompatibles entre sí, al menos de forma directa. También es reveladora la heterogeneidad a nivel técnico dentro de los sistemas. La gran variedad de medios de transmisión y estructuras de control es una muestra de la gran versatilidad de la tecno- logía al entrar en el hogar pero a la vez un nuevo síntoma de falta de estandarización. Por ejemplo, los sistemas estudiados presentan principalmente las siguientes arquitecturas: centralizada (42\%), distribuida (32\%), híbrida (23\%) y decentralizada (12\%). Ante estos datos parece imprescindible una homogenización a nivel sectorial que sirva de garantía tanto a los usuarios como a los propios agentes del sector, ya que la falta de interoperabilidad y estandarización es un escollo para la generación de economías de escala, aspecto clave para el despegue de este tipo de tecnologías.

9. En estrecha relación con el punto anterior, es destacable también la falta de compatibilidad en el hogar digital entre dispositivos y programas de diferentes fabricantes, lo que supone un obstáculo para la realización de proyectos domóticos y la implementación de servicios avanzados en los que se combinen diversas tecnologías. Sin duda, se trata de una barrera que debe de ser superada para encaminarse hacia unos estándares más homogéneos que faciliten el acceso y manejo de los sistemas instalados en el hogar (16). Tal y como apuntan autores como Giménez (17), la flexibilidad de los sistemas que se pretendan introducir en el mercado es clave para su completa operatividad.

10. Otra de las razones apuntadas es el retraso histórico, con respecto otros países europeos o EE.UU. o Canadá, de tradición tecnológica. El menor desarrollo del sector tecnológico y de investigación (18) se refleja en la menor demanda de estos productos en la sociedad. Asimismo, tal y como apuntan estudios como el de Varo et al. (5), el sector empresarial de la construcción tiene un carácter conservador, lo que implica un nivel bajo de inversión en innovaciones de tipo tecnológico.

11. Desde las administraciones públicas no se ha potenciado la industria. Las escasas viviendas de promoción pública que se iniciaron durante los años de la burbuja explica, en parte, este desinterés. Asimismo, y a pesar de que España ha sido un país pionero en la creación de normativa relacionada con el hogar digital, la Administración no ha definido una mínima dotación de tecnología para las viviendas más allá de lo establecido para las infraestructuras comunes de telecomunicación, orientadas fundamentalmente a garantizar el acceso a los servicios de telefonía, televisión e Internet. Por ejemplo, la obligatoriedad de alarmas técnicas contra incendios o escapes de gas ayudaría sin dudas al despegue del sector.

12. Y más allá, ¿̇suponía la domótica una nueva concepción de algo tan tradicional como el hogar? ¿Sería necesario, primero, generalizar una nueva visión de la vida en el hogar? En sentido demográfico, las generaciones que realmente han adquirido su casa en el periodo de máximos (años 2001/2007) pueden no coincidir exactamente con las generaciones realmente tecnológicas que van llegando a edades de emancipación. Además, el profundo cambio demográfico de esta última década pudo plantear problemas de adaptabilidad a un sector cuya capacidad de respuesta no fue tan rápida. Los contextos sociotécnicos de gran complejidad en los que nos vemos inmersos generan nuevas prácticas sociales de gestión de riesgos que no son recibidas por todos los miembros de la sociedad con el mismo grado de confianza (19). Esta confianza se torna clave en la decisión de inversión en sistemas domóticos. 


\section{FUTURO}

Una de las preguntas clave que se plantean de cara al futuro en este sector es si la domótica, tan estrechamente ligada al desarrollo y la innovación, puede llegar al mercado de masas en los momentos de recesión actuales. En este aspecto, hay que tener en cuenta además que la complejidad de la realidad social dificulta la elaboración de predicciones, especialmente cuando uno de los componentes que se manejan tiene carácter tecnológico (20). A pesar de ello, los últimos análisis apuntan de nuevo a un futuro prometedor para la domótica y se vislumbran con cierta claridad algunas claves de futuro que enunciamos a continuación:

El futuro de la domótica y del hogar digital está muy relacionado con dos paradigmas conocidos como el Internet de las cosas y la inteligencia ambiental. El Internet de las cosas vislumbra un hogar plagado de dispositivos conectados a Internet, esto es, un escenario en el que elementos como una mesa o una bombilla tengan conexión a la red. Por otra parte, la inteligencia ambiental (18) aprovecha este nuevo nivel de conectividad para dibujar un futuro en el que el entorno se adapta automáticamente a las necesidades y preferencias de cada usuario. No obstante, el camino todavía es largo hasta llegar hacia ese grado de virtuosismo tecnológico, y probablemente para cuando se llegue la propia idea del hogar digital puede haber cambiado.

Los fabricantes domóticos han aspirado tradicionalmente a convertir su sistema domótico en el centro del hogar digital. El tiempo ha demostrado que la domótica, si existe, es solo un elemento más del hogar. Desde este punto de vista, para el usuario la domótica está más cerca de ser el mando a distancia que la televisión, es decir, proporciona unos servicios que el usuario valora pero no es imprescindible. Por este motivo, el futuro apunta claramente a un usuario que utiliza la domótica desde su teléfono o tableta como una aplicación más.

A finales de la década pasada apareció una nueva generación de dispositivos inalámbricos con importantes aplicaciones en domótica, como Z-wave o Zigbee. Este tipo de sistemas domóticos tienen una fiabilidad similar a la de un sistema cableado y además no requieren de la realización de obras para su instalación, lo que les permite acceder a la totalidad del parque de viviendas. Esto, unido a su bajo precio en comparación con otros sistemas como EIB, es fundamental para que estos sistemas puedan llegar a ser productos de masas.

Pese a que la mayoría de sistemas parten de un enfoque integral de la domótica en el que un mismo sistema proporciona diferentes servicios (por ejemplo: regulación de temperatura, control de iluminación, etc.), la proliferación de sistemas específicos para alguno de estos servicios es un hecho. Por ejemplo, según el estudio de Berg Insight (2), de los 1,06 millones de sistemas domóticos domésticos instalados en Europa en 2012, solo 0,15 millones corresponden con sistemas integrales, es decir, los 0,91 millones restantes se corresponde con sistemas que proporcionan únicamente una de las funcionalidades del hogar digital. Evidentemente, un mismo hogar puede contar con varios de estos sistemas, especialmente si se tiene en cuenta que los teléfonos de última generación y las tabletas proporcionan una interfaz unificada para el acceso a estos diferentes servicios. A pesar de que este enfoque presenta mayores dificultades para la integración real de los servicios, es decir, para proporcionar al usuario servicios basados en más de una de las funcionalidades, presenta la ventaja de permitir al usuario la incorporación paulatina de diferentes funcionalidades al hogar. En este sentido la instalación de dispositivos domóticos en el hogar debe de proveer una unidad de tratamiento, seguimiento y control conjunto que no derive en sensaciones negativas de agobio para el usuario (21).

En los próximos años se incorporarán progresivamente al hogar robots que prestarán servicios domésticos. De hecho, en sentido estricto, también son robots algunos de los electrodomésticos existentes en el hogar desde hace ya décadas tales como los robots de cocina. La integración de estos dispositivos en el hogar digital tiene mucho potencial gracias a los nuevos servicios que surgirán fruto de esta integración. Por ejemplo, en el trabajo de Borja et al. (22) se utiliza un robot de telepresencia como complemento al sistema de seguridad en el hogar y según los mismos autores el propio usuario puede invocar y controlar un aspirador autónomo desde su sistema domótico. Finalmente, se están desarrollando también arquitecturas y protocolos específicos para este tipo de aplicaciones (23).

Sin duda, si hablamos de los habitantes, la tardía emancipación familiar puede facilitar la instalación de sistema domóticas en la rehabilitación de los hogares, ya que las nuevas generaciones están más habituadas al uso de la tecnología pudiendo romper la reticencia de las generaciones más mayores dentro del hogar.

Algunos de los tipos de hogares que se consolidan como el de parejas sin hijos o viviendas unifamiliares de menores de 65 años se perciben más abiertos a los cambios tecnológicos en la vivienda, $y$, en algunos casos con mayor disponibilidad económica para invertir en sistemas de calidad. Las nuevas generaciones, más habituadas a estas tecnologías, tienen mucho que decir en este campo ya han crecido en una sociedad de consumo donde se valora más la simbología del producto que la funcionalidad del mismo (24), aunque sólo si el mercado domótico es capaz de adaptarse a los estándares de manejo de software. Si bien suponemos que estas características de las nuevas generaciones los hace más proclives a la adaptación de sistemas domóticos, en realidad es difícil definir un «perfil de usuario» tipo de tecnología domótica (25), de ahí la imprecisión de muchas predicciones.

Como hemos visto en el punto anterior la adaptabilidad a los nuevos cambios en las características de vivienda se muestran claves en el despegue de la domótica. Si analizamos las potencialidades derivadas de la reestructuración del mercado de la construcción, vemos como la rehabilitación y la posible inversión en viviendas de protección oficial se presentan como dos de los terrenos con mayores posibilidades debido al reducido número de viviendas que previsiblemente se construirá en el futuro próximo como consecuencia de la crisis y del excedente de viviendas vacías.

El mayor porcentaje de construcción de viviendas unifamiliares aisladas junto con los aumentos de superficie en las viviendas unifamiliares, sugieren un mercado potencial al sector. Se puede inferir que el perfil tipo de habitante de esta tipología de viviendas difiere del perfil tipo del habitante de vivienda en bloque, ya que al disponer de una superficie mayor, este tipo de viviendas suelen necesitar una mayor inversión económica. Este cambio en las tipologías de la vivienda 
construidas también ha estado acompañado de cambios en las características internas de las mismas. Con el aumento de las viviendas unifamiliares desde 2007 también ha aumentado su tamaño medio, especialmente hasta el año 2009. Todas las tipologías de viviendas parecen haber frenado su aumento de tamaño en el año 2010 excepto en las viviendas en edificios en bloque que ya habían disminuido su tamaño ligeramente, y en 2010 parece incrementarlo casi imperceptiblemente. Las viviendas pareadas son las que han registrado un mayor aumento de la superficie media en estos últimos años. Estos cambios de dimensiones pueden verse como potenciales necesidades de diferentes sistemas domóticos.

Por su parte, a partir de 2008 se empieza a recuperar la proporción de viviendas de protección oficial tras el descenso durante los años de la burbuja (ver Tabla 1), hasta incluso superar los porcentajes de VPO previos al gran periodo de expansión viviendo por este sector. La inserción de tecnología domótica en viviendas de promoción pública sería un punto clave de la generalización de este sector en el mercado inmobiliario general.

Tabla 1. Porcentaje de viviendas terminadas libres y de calificación definitiva VPO sobre total de viviendas construidas

\begin{tabular}{|c|c|c|}
\hline & Viv. Libre & VPO \\
\hline 2000 & 87,40 & 12,60 \\
\hline 2002 & 92,76 & 7,24 \\
\hline 2004 & 90,31 & 9,69 \\
\hline 2006 & 90,76 & 9,24 \\
\hline 2008 & 89,15 & 10,85 \\
\hline 2010 & 79,32 & 20,68 \\
\hline 2011 & 70,57 & 29,43 \\
\hline 2012 & 63,28 & 36,72 \\
\hline
\end{tabular}

Fuente: Ministerio de Fomento.

En clave nacional, el excesivo parque de viviendas vacías puede ser útil en el corto plazo para la domótica. La implantación de sistemas domóticos y energéticos como valor agregado podría significar un punto diferenciador para la venta de las mismas sin perder de forma excesiva su valor (26).

El ahorro de energía y otros suministros en la vivienda y la creciente concienciación con respecto a la sostenibilidad medioambiental podrían ser clave también para abrir el mercado. En concreto, el mayor nivel de concienciación medioambiental influye en la instalación de sistemas de ahorro y eficiencia energética en los hogares, no sólo en instalaciones posteriores a la construcción, sino en la demanda de las mismas en los procesos de construcción de nuevas viviendas (27). Asimismo, el incremento de precio ocasionado por los sistemas adicionales que deben instalarse puede ser compensado con creces a lo largo del tiempo gracias a la reducción de consumos que conllevan. Por otro lado, la extensión de la gestión desde las viviendas propias a las comunidades de vecinos puede servir como un paso intermedio entre el Hogar Digital y la Ciudad Digital (28).

En todo caso, conviene olvidar que según la clásica teoría de la Pirámide de Maslow, esta necesidad vendrá desde los sectores de población que tengan cubiertas sus necesidades más básicas primero, para ir luego completando a una escala de deseos, posibilidades y expectativas tanto económicas como sociales. Podemos inferir por tanto, que este incremento de la domótica será gradual y debería de diferenciarse según las necesidades de los usuarios para poder adaptarse a su demanda.

Otro tema que merece atención relacionado con el punto anterior son los recientes cambios en la tarificación de la factura eléctrica. El 1 de abril de 2014 entró en vigor el nuevo sistema de cálculo del recibo de la luz, que acerca a los consumidores a la posibilidad de ser facturados por lo que realmente consumen. De este modo, es posible ajustar los patrones de consumo eléctrico con objeto de beneficiarse de los mejores precios horarios de la electricidad. Por tanto, las programaciones horarias propias características de los sistemas domóticos tienen ante sí una interesante oportunidad para contribuir a la reducción de la factura.

Habituados a una saturación de posibilidades, será necesario que las empresas generen las necesidades de consumo para que se despierte cierta curiosidad sobre los productos. En este sentido, la creación de una demanda exige generar nuevas perspectivas ya que el mercado se rige en gran medida por unas necesidades subjetivas que se enmarcan en la potencialidad del «estilo de vida» como eje de las prácticas de consumo (29). Las nuevas necesidades creadas a través de la continua realimentación tecnológica entre los jóvenes y sus hábitos cotidianos (30) se plantean como imprescindibles en las prestaciones del hogar digital tanto presente como futuro.

Las tendencias tecnológicas actuales están formando usuarios que demandan productos actualizados y personalizados, lo que en el caso de la domótica se traducirá en una adaptación de la misma tanto a las características de la vivienda como del entorno social más cercano. En este sentido se han desarrollado incluso modelos econométricos que cuantifican los factores en la decisión final de invertir en nueva tecnología (17). La confianza por parte de los usuarios en las tecnologías es clave para la expansión de la domótica y para ello será necesaria la normalización de las tecnologías en las prácticas diarias (27).

Asimismo, un público cada vez más diverso y acostumbrado a poder elegir entre un abanico amplio de opciones abre necesariamente la puerta hacia nuevos modelos de consumo alejados de los tradicionales medios de masas (31). La carga simbólica de los productos consumidos es un componente más de los mismos, característica clave en la actual sociedad de consumo (32).

Finalmente, una visión de carácter más social frente a otra más tecnológica ayuda a entender que la producción de aparatos tecnológicos per se no tiene por qué significar una apropiación, sin más, de éstos por parte de la sociedad. En el análisis y proyecciones hay que tener en consideración los múltiples procesos de micro-apropiación social de las personas usuarias de la tecnología (31). En este sentido, se trata de averiguar si distintos grupos sociales, además de conocer las nuevas tecnologías y llegar a tenerlas, se apropian, o no, de ellas y las utilizan de una manera efectiva en sus actividades cotidianas (33).

Además, se requiere también una apropiación cultural previa que no está determinada únicamente por las posibilidades implícitas en el uso de la tecnología (34), sino también por el imaginario simbólico de valores, representaciones y prácticas 
compartidas de cada grupo social. Lo que se ha denominado «uso significativo» de las nuevas tecnologías (33), medido a través de «variables de significado» (35) donde no sólo la innovación tecnológica es importante sino el uso efectivo que la sociedad o determinados grupos sociales hacen de ella.

\section{CONCLUSIONES}

Aunque algunas conclusiones se han ido desgranando a lo largo del artículo, recogemos en este punto las principales reflexiones. Uno de los puntos clave que se extraen del análisis del mercado de la domótica es que, si bien el despegue no ha sido el esperado, este mercado sigue siendo un campo lleno de posibilidades y potencialidades tanto a nivel nacional como internacional, necesario si hablamos de una mejora en la calidad de vida y de la sostenibilidad de las viviendas, y por tanto de las ciudades, en el futuro. En cualquier caso, el sector debe aprender de los errores ya señalados, cometidos en una época con una coyuntura muy favorable para su desarrollo. Las claves de futuro aportadas, permiten además bosquejar parte del futuro que está por venir.

La rapidez de los cambios sociales supone también un reto para este sector. La vanguardia de las instalaciones y la velocidad a la que se sucedan los nuevos productos mantendrán la domótica en primer plano. Es necesario que este reto no se convierta en obstáculo. Esta inversión en tecnología pasa por ser una de las pocas salidas a una industria de la construcción profundamente dañada, que depende de la innovación y reinvención de la misma para poder seguir en la brecha.

\section{AGRADECIMIENTOS}

Los autores agradecen a la Junta de Andalucía por la financiación del proyecto Gestión Óptima de Edificios de Energía Cero (referencia P11-TEP-8129) y al Ministerio de Ciencia y Educación por la financiación del proyecto Técnicas de Control Predictivo para la Gestión Eficiente de Micro-Redes de Energías Renovables (referencia DPI2010-21589-Co5-01).

\section{REFERENCIAS}

(1) Aibar, E. (1996). La vida social de las máquinas: origen, desarrollo y perspectivas actuales en la sociología de la tecnología. REIS, (76): 141-170, doi: http://dx.doi.org/10.2307/40183990.

(2) Berg Insight. (2013). Smart Homes and Home Automation, 2nd edition.

(3) BSRIA. (2010). Smart Home 2010 Europe Study.

(4) MinT-CASADOMO. (2008). Estudio mint-casadomo 20o8: Sistemas de domótica y seguridad en viviendas de nueva promoción. Madrid: Casadomo Soluciones, SL.

(5) Varo, R., Arroyo, M. A., Sanz, M. D. (2008). Hogar Digital. Presente y futuro del hogar digital. Una visión desde Andalucía. Andalucía: Eticom y Colegio Oficial Ingenieros de Telecomunicación.

(6) Telefónica. (2003). Libro Blanco del Hogar Digital y las Infraestructuras Comunes de Telecomunicaciones. Madrid: Telefónica de España, S.A.

(7) García-Montalvo, J. (2003). La vivienda en España: desgravaciones, burbujas y otras historias. Perspectivas del sistema financiero Español, (78): 1-43.

(8) Nóvoa-Arechaga, I., López-Suárez, P. A. (2007). El mercado de la vivienda en España: una década prodigiosa (19952005). En Decisiones basadas en el conocimiento y en el papel social de la empresa. XX Congreso Anual de AEDEM. Vol. 2.

(9) Balmaseda, M., San Martin, I., Sebastian, M. (2002). Una aproximación cuantitativa a la burbuja inmobiliaria. En Situación Inmobiliaria, (pp. 22-28). Madrid: Servicio de Estudios del Banco Bilbao Vizcaya.

(10) Institut Cerdá, cEDOM, CASADOMO. (2003). Proyecto Prohome: Informe C1. Plan estratégico para el avance de la sociedad de la información en el ámbito doméstico.

(11) García-Montalvo J. (2008). El sector inmobiliario español a principios del siglo XXI: entre la demografía y las expectativas. Clm. Economía, (11): 57-79.

(12) Martín, H., Sáez, F. (2006). Domótica: un enfoque sociotécnico. Madrid: Fundación Rogelio Segovia para el Desarrollo de las Telecomunicaciones.

(13) Hoekstra, J., Vakili-zad, C. (2011). High vacancy rate and rising house price: the Spanish paradox. Tijdschrift voor Economische (Sociale Geografie), 102(1): 55-71, doi: http://dx.doi.org/10.1111/j.1467-9663.2009.00582.x.

(14) CEDOM. (2011). Estudio СЕDOM 2011: Tendencias del mercado español de domótica e inmótica. Asociación Española de Domótica.

(15) Institut Cerdá, CEDOM, CASAdomo. (2004). Proyecto Mercahome: Informes B1, B2 y B3. Ministerio de Industria, Turismo y Comercio.

(16) Ferreira, M. (2004). Camino al hogar inteligente. Informes de la Construcción, 56(494): 5-10, doi: http://dx.doi. org/10.3989/ic.2004.v56.i494.443.

(17) Giménez, G. (2004). La inversión en tecnología: una modelización del proceso de toma de decisiones de consumo. Atlactic Review of Economics, 3(7).

(18) Chaparro, J. (2003), Domótica: la mutación de la vivienda. Scripta Nova. Revista electrónica de geografía y ciencias sociales, $7(146 / 136)$.

(19) García, A., Moles, R.J., Palmen, R., Fàbregues, S. (2009). Reconsiderando la gestión de riesgos en entornos sociotécnicos. Papers, 93:103-126

(20) Bouwman, H., Haaker, T., De Reuver, M. (2012), Some reflections on the high expectations as formulated in the Internet Bubble era. Futures, 44(5): 420-430, doi: http://dx.doi.org/10.1016/j.futures.2012.03.004. 
(21) Recuero, A. (1999). Estado actual y perspectivas de la domótica. Informes de Construcción, 50(459): 9-21, doi: http:// dx.doi.org/10.3989/ic.1999.v50.i459.827.

(22) Borja, R., de la Pinta, J. R., Álvarez, A., Maestre, J. M. (2013) Integration of service robots in the smart home by means of UPnP: A surveillance robot case study. Robotics and Autonomous Systems, 61(2): 153-160, doi: http://dx.doi. org/10.1016/j.robot.2012.10.005.

(23) González-Alonso, I., Álvarez, O., Alonso, A., Gómez, P., Maestre, J.M., García-Fuentes, A. (2012), Towards a new open communication standard between homes and service robots, the DHCompliant case. Robotics and Autonomous Systems, 60(6): 889-900, doi: http://dx.doi.org/10.1016/j.robot.2012.01.006.

(24) Páramo, D. (2006). El fenómeno de consumo y el consumo en marketing. Revista Convergencia, 11(34): 221-250.

(25) Flórez de la Colina, M.A. (2004). Hacia una definición de la domótica. Informes de la Construcción, 56(494): 11-17, doi: http://dx.doi.org/10.3989/ic.2004.v56.i494.444.

(26) Pedreño, A., Ramón, A. B., Moreno, L. (2011). El camino hacia la nueva construcción. Sostenibilidad, innovación y vanguardia. Metode, (68): 74-79.

(27) García-S. Matachana, E., López-Peñalver, F. (2004). El Estado del arte de la tecnología al servicio de la construcción. Informes de la construcción, 56(494): 19-26, doi: http://dx.doi.org/10.3989/ic.2004.v56.i494.445.

(28) Lorente, S., Vinagre, J. J., Rueda, J. I. (2008). La comunidad digital de vecinos. Madrid: Creaciones Copyright.

(29) García-Ruiz, P. (2009). El concepto de "reflexividad" en la sociedad del consumo: algunas propuestas. RES, 12 : 85-102.

(30) Seni, G. (2011). Jóvenes, medios y consumo. Encuentros, 9(2): 13-32.

(31) Bélanger, P.C., Ross, P. (2000). Usos y percepciones de las nuevas tecnologías e incidencias en la vida familiar. Comunicar, 14: 69-78.

(32) Conde, F., Alonso, L.E. (2002). Gente conectada: la emergencia de la dimensión fática en el modelo de consumo glocal. Política y Sociedad, 39(1):27-51.

(33) Echeverría, J. (2008a). Apropiación social de las tecnologías de la información y la comunicación. Revista CTS, 4(10). http://oeibolivia.org/files/Volumen \%204 \%20- \%20N \%C3 \%BAmero \%2010/dosso7.pdf

(34) Sagástegui, D. (2005). Apropiación social de la tecnología: un enfoque sociocultural del conocimiento. Razón y Palabra, 49. http://www.razonypalabra.org.mx/anteriores/n49/bienal/Mesa\%2012/DianaSagastegui.pdf

(35) Toboso, M., Estévez, B. (2012). Propuesta de un sistema de indicadores de apropiación social de tecnologías y su relación con dinámicas de innovación social. En Apodaka, E., Merino, L., Villarreal, M. (Eds.). Crisis y mutaciones de la expertise. Escenarios, políticas y prácticas del conocimiento experto (pp. 173-187). ASCIDE: Zarautz (Guipuzkoa). 